\title{
SPLIT RANK AND SEMISIMPLE AUTOMORPHISM GROUPS OF G-STRUCTURES
}

\author{
ROBERT J. ZIMMER
}

\section{Introduction}

This paper is a continuation of the investigation begun in [1], [3], [4] concerning the semisimple automorphism groups of $G$-structures on compact manifolds. In those papers we were concerned with semisimple groups that preserve a structure which is algebraic and which defines a volume density, i.e. where the structure group $G$ is an algebraic subgroup of $\operatorname{SL}^{\prime}(n, \mathbb{R})$, the matrices with $\mid$ det $\mid=1$. (For higher order structures we assumed that $G$ is an algebraic subgroup of $\operatorname{SL}^{\prime}(n, \mathbb{R}) \cap \mathrm{GL}(n, \mathbb{R})^{(k)}$, the latter being the group of $k$-jets at 0 of diffeomorphisms of $\mathbb{R}^{n}$ fixing the origin.) One of the basic conclusions in the above papers is that for any simple noncompact Lie group $H$ preserving such a $G$-structure, we must have that $H$ locally embeds in $G$. (In fact a stronger assertion is proven. See the above papers and Theorem 2 below.) The main goal of the present paper is to consider the situation in which $H$ is no longer assumed to define a volume density. In this case natural examples easily show that one cannot expect a local embedding of $H$ in $G$. However, our main result asserts that a basic structural invariant of $H$ must be visible in $G$. More precisely, we prove:

Theorem 1. Let $\mathrm{H}$ be a semisimple Lie group with finite center and suppose that $H$ acts smoothly on a compact manifold $M$ so as to preserve $a$ G-structure on $M$, where $G$ is a real algebraic group. Then $\mathbb{R}-\operatorname{rank}(H) \leqslant \mathbb{R}-\operatorname{rank}(G)$.

We recall that the $\mathbb{R}$-rank, or split rank, of a real algebraic group is the maximal dimension of an algebraic torus that is diagonalizable over $\mathbb{R}$. For a semisimple Lie group $H, \operatorname{Ad}(H)$ will be the connected component of the identity of a real algebraic group, and the $\mathbb{R}$-rank, or split rank, of $H$ is defined to be the split rank of this real algebraic group. We shall also clear up

Received June 13, 1986. The author's research was partially supported by a National Science Foundation Grant. 
a point that was left open in [1], [3] concerning the case in which $G$ defines a volume density. Namely, the general results in [3] were established for noncompact simple groups, not for semisimple groups. In [3], a special argument was given that clarified the semisimple situation for the case of Lorentz structures. Here we observe that a simple argument enables us to extend the results of [3] to the semisimple case in general, at least in the case of finite center.

Theorem 2 (cf. [1], [3], [4]). Let $H$ be a connected semisimple Lie group with finite center and no compact factors, and suppose that $H$ acts on a compact $n$-manifold preserving a $G$-structure, where $G$ is algebraic and defines a volume density. Then there is an embedding of Lie algebras $\mathfrak{h} \rightarrow \mathfrak{g}$. Furthermore, the representation $\mathfrak{h} \rightarrow \mathfrak{g} \rightarrow \mathfrak{s} \mathfrak{l}(n, \mathbb{R})$ contains $\operatorname{ad}_{\mathfrak{h}}$ as a direct summand.

Part of this work was done while the author was a visitor at Harvard University. We would like to thank the members of that department for their hospitality.

\section{Preliminaries}

We establish here some preliminary information we shall need for the proofs of Theorems 1 and 2.

Proposition 3. Let $H$ be a connected semisimple Lie group with finite center, acting smoothly on a connected manifold $M$, and assume $p \in M$ is a fixed point. Let $\pi: H \rightarrow \operatorname{GL}\left(T M_{p}\right)$ be the corresponding representation at $p$. If $\pi$ is trivial, then $H$ acts trivially on $M$.

Proof. Let $K \subset H$ be a maximal compact subgroup. It suffices to see that $K$ acts trivially. For a compact group, any smooth action can be linearized around fixed points, so the set of invariant frames for the tangent bundle is both open and closed.

Proposition 4. Suppose $H$ is a connected semisimple Lie group with finite center, acting smoothly on a connected manifold $M$. If the set of fixed points has positive measure, then $H$ acts trivially.

Proof. If the set of fixed points, $F$, has positive measure, choose a density point $p$ for $F$ in the sense of Lebesgue. Then any small ball around $p$ intersects $F$ in a set of positive measure. The action of the maximal compact subgroup $K \subset H$ can be linearized around $p$, which implies that $K$ leaves a set of vectors in $T M_{p}$ invariant which has positive measure in $T M_{p}$. It follows that this linear representation of $K$ is trivial, and the proof of Proposition 3 completes the proof.

If a Lie group $H$ acts smoothly on a manifold $M$, and $m \in M$, we let $H_{m}$ be the stabilizer of $m$ in $H$, and $\mathfrak{h}_{m} \subset \mathfrak{h}$ the Lie algebra of $H_{m}$. If $V$ is a vector space we let $\mathrm{Gr}_{d} V$ ) be the Grassman variety of $d$-dimensional linear subspaces. 
For a Lie group $L$, we let $L^{0}$ be the identity component. If a Lie group $L$ is the identity component of an algebraically connected real algebraic group, by a rational homomorphism of $L$ into a real algebraic group we mean the restriction of (a necessarily unique) rational homomorphism of the ambient algebraic group. The following is standard.

Lemma 5. Suppose $H$ is a Lie group acting smoothly on a manifold $M$. Let $d$ be the minimal dimension of an $H$-orbit in $M$. Then $M_{1}=\{m \in M \mid \operatorname{dim}(G m)=$ $d$ \} is closed, and the map $m \rightarrow \mathfrak{h}_{m}$ defines a continuous map $\varphi: M_{1} \rightarrow \mathrm{Gr}_{q}(\mathfrak{h})$, where $q=\operatorname{dim}(H)-d$. Further, $\varphi$ is an H-map, where $H$ acts on $\operatorname{Gr}_{q}(\mathfrak{h})$ via $\operatorname{Ad}(H)$.

We recall briefly the notion of the algebraic hull of a cocycle defined for an ergodic group action (see [4] or [2] for an elaboration). Suppose that $H$ is a locally compact group acting ergodically on a standard measure space $(M, \mu)$. Suppose that $G$ is a real algebraic group and that $\alpha: H \times M \rightarrow G$ is a cocycle, i.e., the following identity is satisfied (for each $h_{1}, h_{2} \in H$, and almost all $m \in M): \alpha\left(h_{1} h_{2}, m\right)=\alpha\left(h_{1}, h_{2} m\right) \alpha\left(h_{2}, m\right)$. We recall that two cocycles $\alpha, \beta$ are called equivalent if there is a measurable $\varphi: M \rightarrow G$ such that for each $h$ and almost all $m, \beta(h, m)=\varphi(h m)^{-1} \alpha(h, m) \varphi(m)$.

Lemma 6 ([2], [4], [5]). There is an algebraic subgroup $L \subset G$ with the following properties:

(i) $\alpha$ is equivalent to a cocycle taking all its values in $L$.

(ii) For any proper algebraic subgroup $L^{\prime} \subset L, \alpha$ is not equivalent to a cocycle taking all its values in $L^{\prime}$.

(iii) $U p$ to conjugacy in $G, L$ is the unique algebraic subgroup satisfying (i), (ii).

(iv) If $\alpha$ is equivalent to a cocycle taking all its values in some closed subgroup $L_{0} \subset G$, then some conjugate of $L_{0}$ is contained in $L$.

$L$ is then called the algebraic hull of $\alpha$, and it is well defined up to conjugacy in $G$. The following property is easily established.

Lemma 7. Suppose $p: G_{1} \rightarrow G_{2}$ is a rational homomorphism of real algebraic groups. If $\alpha$ is a $G_{1}$-valued cocycle with algebraic hull $L_{1}$, then the algebraic hull of the $G_{2}$-valued cocycle $p \circ \alpha$ is the algebraic hull of $p\left(L_{1}\right)$ (in which, we recall, $p\left(L_{1}\right)$ is a subgroup of finite index).

\section{Proof of Theorem 1}

Let $M_{1}$ be as in Lemma 5. Since $M_{1}$ is a compact $H$-space, we can choose a minimal $H$-space $M_{0} \subset M_{1}$, i.e., a closed $H$-invariant subset in which every orbit is dense. Then, letting $\varphi$ be as in Lemma 5 as well, we have that $\varphi\left(M_{0}\right) \subset \mathrm{Gr}_{q}(\mathfrak{h})$ is minimal. However, the action of $H$ on $\mathrm{Gr}_{q}(\mathfrak{h})$ is algebraic, and hence every orbit is locally closed. It follows that $\varphi\left(M_{0}\right)$ consists of a 
single compact $H$-orbit. Fix $x \in M_{0}$, and let $\mathfrak{h}_{x}=\mathfrak{h}_{0}$. Then we can consider $\varphi$ as an $H$-map $\varphi: M_{0} \rightarrow \operatorname{Ad}(H) / N\left(\mathfrak{h}_{0}\right) \subset \mathrm{Gr}_{q}(\mathfrak{h})$, where $N\left(\mathfrak{h}_{0}\right)$ is the normalizer of $\mathfrak{h}_{0}$ in $\operatorname{Ad}(H)$. In particular, the algebraic subgroup $N\left(\mathfrak{h}_{0}\right)$ is cocompact in $\operatorname{Ad}(H)$, and therefore we can find a maximal $\mathbb{R}$-split torus $T$ of $\operatorname{Ad}(H)$, with $T \subset N\left(\mathfrak{h}_{0}\right)$.

Let $\mathfrak{n}$ be the Lie algebra of $N\left(\mathfrak{h}_{0}\right)$, so that $\mathfrak{h}_{0} \subset \mathfrak{n}$ is an ideal. The adjoint representation yields a rational (and in particular semisimple) representation $T \rightarrow \mathrm{GL}\left(\mathfrak{h} / \mathfrak{h}_{0}\right)$. Let $T_{0} \subset T$ be the kernel, so that $T_{0}$ is an $\mathbb{R}$-split subtorus. Since the representation of $T_{0}$ on $\mathfrak{h}$ is semisimple, we can write $\mathfrak{h}=\mathfrak{h}_{0} \oplus W$, where $W \subset \mathfrak{h}$ is a subspace and $T_{0}$ acts trivially on $W$. In particular, $\mathfrak{h}_{0}$ contains all the root spaces of $T_{0}$ acting via $\mathrm{Ad}_{H}$ on $\mathfrak{h}$ corresponding to nontrivial roots. The algebra generated by the nontrivial root spaces for $T_{0}$ is an ideal, and hence $\mathfrak{h}_{0}$ contains an ideal of $\mathfrak{h}$, say $\mathfrak{h}_{1}$, containing all the nontrivial root spaces for $T_{0}$. Thus we can write $\mathfrak{h}$ as a sum of ideals, $\mathfrak{h}=\mathfrak{h}_{1} \oplus \mathfrak{h}_{2}$, where $T_{0}$ acts trivially on $\mathfrak{h}_{2}$. Since $\mathfrak{h}_{2}$ is semisimple, it follows that $\mathrm{t}_{0} \subset \mathfrak{h}_{1} \subset \mathfrak{h}_{0}$. Let $H_{1}$ be the connected normal subgroup of $H$ corresponding to $\mathfrak{h}_{1}$. Then $\mathfrak{h}_{1}$, and hence $H_{1}$, acts trivially on $\mathfrak{h} / \mathfrak{h}_{0}$, and by Proposition 3, $H_{1}$ acts locally faithfully on $T(M)_{x} / T(H x)_{x}$. In particular, $T_{0}$ acts rationally and locally faithfully on $T(M)_{x} / T(H x)_{x}$. Let $T_{1}$ be a split torus complementary to $T_{0}$ in $T$. We then have that $T=T_{0} \times T_{1}$, and $T_{1}$ acts faithfully on $\mathfrak{h} / \mathfrak{h}_{0}$.

Now let $M_{2} \subset M_{0}$ be a minimal $N\left(\mathfrak{h}_{0}\right)^{0}$ space. Since $\left(H_{x}\right)^{0}$ is normal in $N\left(\mathfrak{h}_{0}\right)$, it fixes all points of $N\left(\mathfrak{h}_{0}\right) x$, and hence fixes all points in the closure of this orbit, in particular all points in $M_{2}$. Since the dimension of all stabilizers in $H$ of points in $M_{0}$ are the same, we have $\mathfrak{h}_{m}=\mathfrak{h}_{0}$ for all $m \in M_{2}$. Thus for $m \in M_{2}$, we can identify the tangent space to the $H$-orbit through $m$ with $\mathfrak{h} / \mathfrak{h}_{0}$. The representation of $H_{1}$ on $T(M)_{m} / T(H m)_{m}$ will vary continuously over $m \in M_{2}$, and since $H_{1}$ is semisimple and $M_{2}$ is connected, all these representations are equivalent. In particular, the representations of $\left(T_{0}\right)^{0}$ on these spaces are all rational, and all equivalent.

Choose a probability measure on $M_{2}$ which is invariant and ergodic under $T^{0}$ [2, Chapter 4]. Let $\alpha: T^{0} \times M_{2} \rightarrow \mathrm{GL}(n, \mathbb{R})$ be a cocycle corresponding to the action of $T^{0}$ on the tangent bundle of $M$ over the space $M_{2}$ (cf. [4]). Let $L$ be the algebraic hull of this cocycle. Since $H$, and in particular $T^{0}$, leaves a $G$-structure on $M$ invariant, we have (up to conjugation) $L \subset G$. By our observations above, we can measurably trivialize $T M$ over $M_{2}$ in such a way that $T M \cong M \times \mathbb{R}^{n}, \mathbb{R}^{n}=V_{1} \oplus V_{2}, V_{1} \cong \mathfrak{h} / \mathfrak{h}_{0}$, such that for $t \in T_{0}^{0}$, we have

$$
\alpha(m, t)=\left(\begin{array}{cc}
I & 0 \\
0 & \pi_{2}(t)
\end{array}\right),
$$


where $\pi_{2}$ is a faithful rational representation, and for $t \in T_{1}^{0}$, we have

$$
\alpha(m, t)=\left(\begin{array}{cc}
\pi_{1}(t) & * \\
0 & *
\end{array}\right),
$$

where $\pi_{1}(t)$ is $\operatorname{Ad}(t)$ acting on $\mathfrak{h} / \mathfrak{h}_{0}$, and, as we remarked above, is a faithful rational representation. Let $\beta$ be the projection of $\alpha$ in $\operatorname{GL}\left(V_{1}\right) \times \mathrm{GL}\left(V / V_{1}\right)$. To prove the theorem, it suffices to see that the split rank of $L$ is at least as large as $\operatorname{dim}(T)$, and by Lemma 7 , to prove this it suffices to see that the split rank of the algebraic hull of $\beta$ is at least $\operatorname{dim}(T)$. Thus, we need only see that if $\pi$ is a faithful rational representation of $T^{0}$, then the algebraic hull of the cocyle $\beta(m, t)=\pi(t)\left(m \in M_{2}\right)$ is locally isomorphic to $T$. Let $T^{*}$ be the algebraic hull of the group $\pi\left(T^{0}\right)$; then $T^{*}$ is a split torus, $\pi\left(T^{0}\right) \subset T^{*}$ is of finite index, and $\operatorname{dim}(T)=\operatorname{dim}\left(T^{*}\right)$. If $\beta$ is equivalent to a cocycle into $Q \subset T^{*}$, then there is a measurable $T^{0}$-map $\varphi: M_{2} \rightarrow T^{*} / Q$. Since there is a finite $T^{0}$-invariant measure on $M_{2}$, there is one on $T^{*} / Q$ as well, and if $Q$ is algebraic, it is clear that $\operatorname{dim}(Q)=\operatorname{dim}(T)$. This complete the proof.

\section{Proof of Theorem 2}

The argument of [1, Lemma 6], using the Borel density theorem, shows that the Lie algebra of the stabilizer of almost every point is an ideal. By Proposition 4 , it follows that almost every point has a discrete stabilizer. The proof then follows as in the simple case, as in [3] or [4].

\section{References}

[1] R. J. Zimmer, Semisimple automorphism groups of G-structures, J. Differential Geometry 19 (1984) 117-123.

[2] __ Ergodic theory and semisimple groups, Birkhauser, Boston, 1984.

[3] _ On the automorphism group of a compact Lorentz manifold and other geometric manifolds, Invent. Math. 83 (1986) 411-426.

[4] Er _ Ergodic theory and the automorphism group of a G-structure, Proc. Conf. in honor of G. W. Mackey, Math. Sci. Res. Inst. Publ., Springer, Berlin, to appear.

[5] __. An algebraic group associated to an ergodic diffeomorphsm, Compositio Math. 43 (1981) 59-69.

UNIVERSITY OF CHICAGo 
